\begin{tabular}{|l|l|l|l|}
\hline Eiszeitalter $u$. Gegenwart & $\mathbf{3 0}$ & $\begin{array}{c}221-228 \\
5 \mathrm{Abb} .\end{array}$ & Hannover 1980 \\
\hline
\end{tabular}

\title{
Neue Meeresspiegeldaten aus dem Raum Wangerooge
}

\author{
JöRG HANISCH *)
}

Sea level, radiometric dating, Commelinidea (Phragmetis communis), Venerida (Scobicularia plana), bock marsch sediment, core cuttings, Holocene

North Sea (Wangerooge), North-West German Lowlands, Niedersachsen. TK 25 Nr.: 2213

$\mathrm{K}$ u r z f a s s ung: Wurzeln und Stengel von Phragmites communis aus Brackwasser-Sedimenten, die etwa $6 \mathrm{~km}$ nördlich Wangerooge in 23,1 bis 24,4 m unter NN erbohrt wurden, brachten ${ }^{14} \mathrm{C}$-Alter zwischen 7500 und 8000 v.h. Danach lag der Hochwasserspiegel der Nordsee in diesem Zeitraum etwa 24 m unter NN.

Datierungen von in Lebendstellung entnommenen Muschelklappen von Scrobicularia plana ergeben für die Zeit um 1500 v.h. eine Hochwassermarke von ca. 1,3 m unter der heutigen.

Für die Zeit um 550 v.h. kann mit Hilfe von Gräsern und Wurzeln eines ehemaligen Strandnelkenrasens sowie von Sedimentstrukturen einer Watt- bis Grodensedimentabfolge ein MTHW an der deutschen Nordseeküste angegeben werden, das dem heutigen entspricht.

\section{[New Sea Level Data from the Region of Wangerooge]}

A bst r a c t: Remnants of Phragmites communis from brackwater sediments now at deth of 23.1 to $24.4 \mathrm{~m}$ below NN about $6 \mathrm{~km}$ north of Wangerooge were dated at 7500 to 8000 B.P. According to this the mean high water level in the North Sea lay at about $24 \mathrm{~m}$ below NN during this time interval.

Radiocarbon dating of shells of Scrobicularia plana which were found in living position in fossil tidal flat sediments indicates a high water level at about 1500 B.P. of $1.3 \mathrm{~m}$ below the present one.

About 550 B.P. the mean high water level on the German North Sea coast was practically the same as today. This is deduced from radiometric, botanic, and sedimentary studies of former tidal flat to salt marsh deposits.

\section{Einführung}

Im Rahmen der geologischen Untersuchungen des Nieders. Landesamtes f. Bodenforschung (NLfB) über die Ursachen des Strand- und Dünenabtrags auf der Insel Wangerooge wurden Schilfstengel und -wurzeln aus einer Brackwasserablagerung aus dem heutigen Seegebiet nördlich der Insel, Muschelklappen aus einer alten Wattschicht und alte Grodensedimente radiometrisch untersucht. Die Datierungen erfolgten im ${ }^{14} \mathrm{C}$-Labor des NLfB (Leitung: M. A. GeYH). Sie lieferten drei wichtige Zeitmarken zur Entstehung und Verlagerung Wangerooges, sind aber gleichzeitig für das Meeresspiegel-Anstiegsgeschehen in der Deutschen Bucht von Bedeutung. Die pollenanalytische Auswertung der Brackwasser- und Grodenproben (durch K. J. Meyer, NLfB) gab wertvolle Hinweise auf das jeweilige Ablagerungsniveau im Bezug auf die damaligen Hochwassermarken.

\section{Meeresspiegelindikatoren}

Drei verschiedenartige Fazieseinheiten, die im Raum Wangerooge in unterschiedlicher Tiefenposition auftreten, können als Meeresspiegelanzeiger für das fossile Mitteltidehochwasser herangezogen werden. ${ }^{14} \mathrm{C}$-Altersdatierungen an Brackwassersedimenten aus der heutigen offshore-Zone der Insel sowie an Watt- und Grodensedimenten vom Nordstrand Wangerooges liefern drei neue Zeit-Höhen-Marken des Transgressionsgeschehens.

*) Anschrift des Verfassers: Dr. J. Ha n is ch, Bundesanstalt für Geowissenschaften und Rohstoffe, Stilleweg 2, D-3000 Hannover 51. 


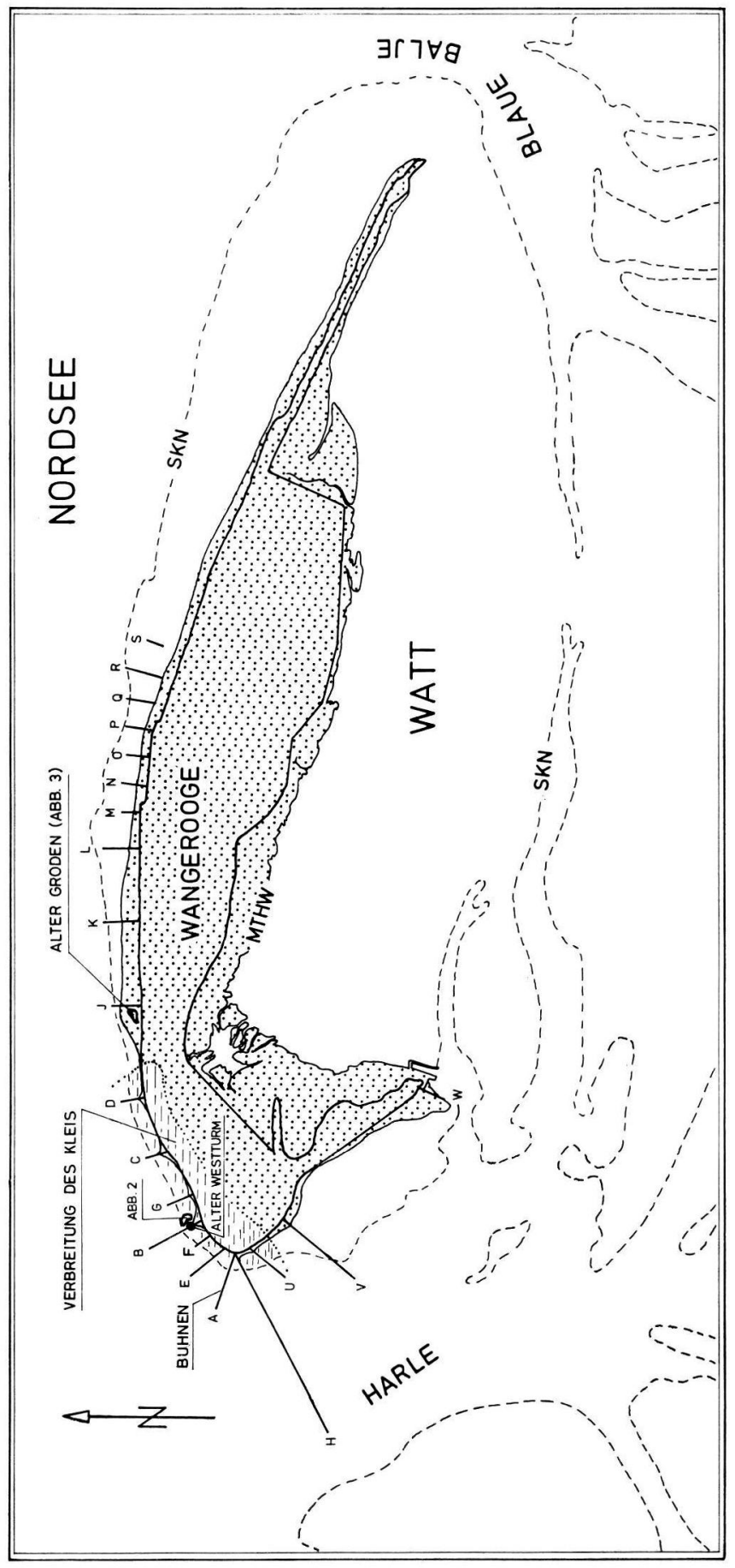

Abb. 1: Ubersichtskarte der Insel Wangerooge. 
2.1. Brackwassersedimente aus der offshore-Zone

Von insgesamt 31 Bohrungen, die im Seegebiet nördlich von Wangerooge abgeteuft wurden, traf als einzige Bohrung A 10 (ca. $6 \mathrm{~km} \mathrm{~N}$ der Insel, Position 55 $51,4^{\prime} \mathrm{N} /$ $07^{\circ} 50,7^{\prime} \mathrm{E}$ ) holozäne Brackwasser-Sedimente an. Diese lagen unter nur 0,67 m marinem Sand und wurden in einer Mächtigkeit von 2,13 m erbohrt, wobei die Basis der brackischen Ablagerungen nicht erreicht wurde.

Die gesamte Abfolge enthält Bestandteile eines marinen Plankton (ForaminiferenInnenschalen, Hystricho-Sphaeriden) sowie Süßwasserelemente wie Zellkolonien der Grünalge Pediastrum.

Aus den feinklastischen Sedimenten ausgelesene und ausgeschlämmte Reste von Phragmites communis (Wurzeln und Stengel) ergaben folgende ${ }^{14} \mathrm{C}$-Alter:

Tiefenintervall ( $\mathrm{m}$ unter $\mathrm{NN}$ ) $\quad \mathrm{Hv}$-Nummer $\quad{ }^{14} \mathrm{C}$-Alter v.h.

$\begin{array}{lll}23,08-23,24 & 8600 & 7540 \pm 80 \\ 23,48-23,73 & 8601 & 7980 \pm 60 \\ 24,07-24,39 & 8602 & 7960 \pm 205\end{array}$

Die Umrechnung der gemessenen Wassertiefen auf NN wurden dankenswerter Weise vom Deutschen Hydrographischen Institut, Hamburg, vorgenommen. Die von LudwIG et al. (1979) veröffentlichten SKN-Werte für die Proben aus Bohrung A 10 sind nach freundlicher mündlicher Mitteilung von $\mathrm{H}$. STREIF mit einem systematischen Fehler behaftet.

Nach ökologischen Studien von Scheer (1953) kann Phragmites communis heute in der Brackwasser- und Gezeitenzone nur zwischen $26 \mathrm{~cm}$ unter und $72 \mathrm{~cm}$ über dem MTHW-Niveau existieren. Ausgehend von diesem ökologischen Befund kann von der Entnahmehöhe auf einen minimal und maximal möglichen Hochwasserstand geschlossen werden. Bei Annahme einer Probenentnahme an der untersten Grenze des ökologischen Bereichs ergibt dies ein maximal $26 \mathrm{~cm}$ d a r über liegendes, früheres MTHW. Nimmt man eine Entnahmehöhe von der oberen, durch Phragmites communis angezeigten Marge an, resultiert ein maximal $72 \mathrm{~cm}$ d a r u n t e r liegendes, früheres MTHW.

Probe 8600 wurde aus einer mittleren Tiefe von $23,16 \mathrm{~m}$ unter NN entnommen. Das damalige MTHW lag also maximal $26 \mathrm{~cm}$ oberhalb von $-23,16 \mathrm{~m} \mathrm{NN}(=-22,9 \mathrm{~m} \mathrm{NN})$ und maximal $72 \mathrm{~cm}$ unterhalb der Entnahmehöhe, also 23,88 m unter NN. Dasselbe gilt für die übrigen beiden Proben entsprechend. Zusammen mit den Datierungsfehlerintervallen ergeben diese maximalen und minimalen Höhenmarken Rechtecke, die als indikative Bereiche der Proben bezeichnet werden können (Abb.2) Wie ersichtlich, ist das Fehlerintervall bei Probe Hv 8602 im Vergleich zu den beiden anderen Proben groß. Daraus erklärt sich das scheinbar geringere Alter von $\mathrm{Hv} 8602$ zu Hv 8601, obwohl die Profilabfolge das Gegenteil zeigt. Durch das Fehlerintervall von Probe Hv 8601 wird jedoch der mögliche Datierungsfehler von $\mathrm{Hv} 8602$ eingeengt (Abb. 2).

Durch Verbinden der äußersten Ecken der Rechtecke in Abb. 2 wird die Marge angezeigt, innerhalb der sich mögliche Meeresspiegel-Änderungen vollzogen haben. Für die Meeresspiegel-Anstiegskurve ergeben sich so minimale und maximale Steigungen (Abb. 2). Der kleinstmögliche Anstieg betrug zwischen 8165 v.h. und 7460 v.h. nur $10 \mathrm{~cm}$, der größtmögliche zwischen 7920 v.h. und 7620 v.h. 1,42 m (von 24,32 auf 22,90 m unter NN). Der wahrscheinliche Anstieg des MTHW zwischen etwa 8000 v.h. und 7500 v.h. hat sich jedoch von 24,1 auf 23,15 unter NN vollzogen (Abb. 2). Dies entspräche einem Anstieg von ca. $19 \mathrm{~cm}$ pro Jahrhundert, wenn man Setzung unberücksichtigt läßt. 


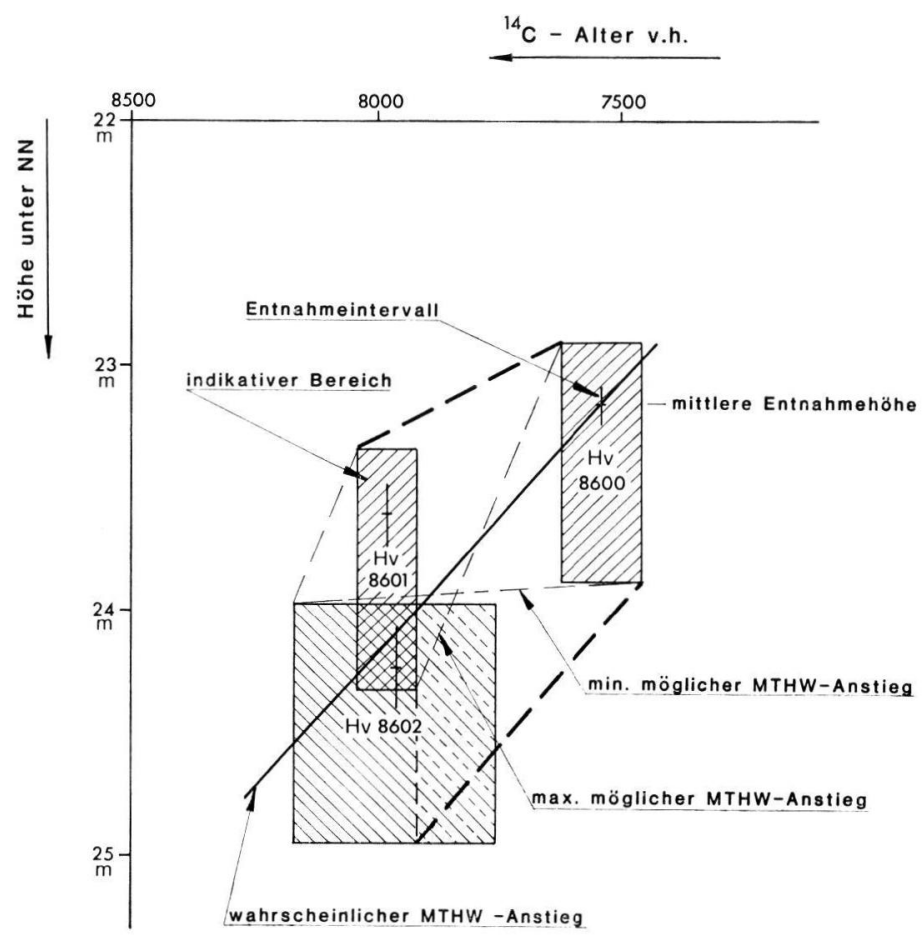

Abb. 2: Aussagekraft der Proben Hv 8600, 8601 und 8602 auf die früheren Hochwasserstände.

Gesichert ist durch diese drei Daten jedoch nur ein früherer mittlerer Hochwasserstand von ca. $-24 \mathrm{~m} \mathrm{NN}$, da der minimal mögliche Anstiegsbetrag von $10 \mathrm{~cm}$ innerhalb der meßtechnischen Fehlergrenzen liegt.

2.2. Muschelklappen ausder Klei-Schicht beim Alten West turm Wangerooges

Bedingt durch die fortschreitende Stranderniedrigung im Westteil von Wangerooge wird, speziell im Bereich der Buhnen und Dünenschutzwerke, immer häufiger eine steife, sandige, etwa $60 \mathrm{~cm}$ mächtige Schlickschicht durch Sturmfluten freigelegt. Diese Klei-Lage, im Volksmund „Knickschicht" genannt, hat nach SINDOwsKI (1969) flächenmäßig eine Verbreitung, die von etwa Buhne D bis vor das Westende der Insel reicht (Abb. 1). Direkt östlich der Buhne B ist das oberste Viertel dieser sandig-siltigen Tonschicht über viele Quadratmeter von Mollusken der Gattungen Scrobicularia plana und Cardium edule durchsetzt. Diese befinden sich größtenteils in Lebendstellung (Abb. 3).

Um eine mögliche Kontaminierung durch heutiges, im Meerwasser gelöstes $\mathrm{CO}_{2} \mathrm{zu}$ umgehen, wurden die zur Altersdatierung herangezogenen Scrobicularien aus einer Zone entnommen, in der sie noch durch weitgehend impermermeablen Schlick überdeckt waren. Die Datierung der Schalen ergab ein ${ }^{14} \mathrm{C}$-Alter von $1540 \pm 75$ v.h. (Hv 9257). Zusammen mit einer früheren Datierung (SINDOwSKI 1969: Hv 300, $1450 \pm 180$ v.h.) resultiert ein mittleres Alter der beiden Proben von $1525 \pm 70$ v.h. 

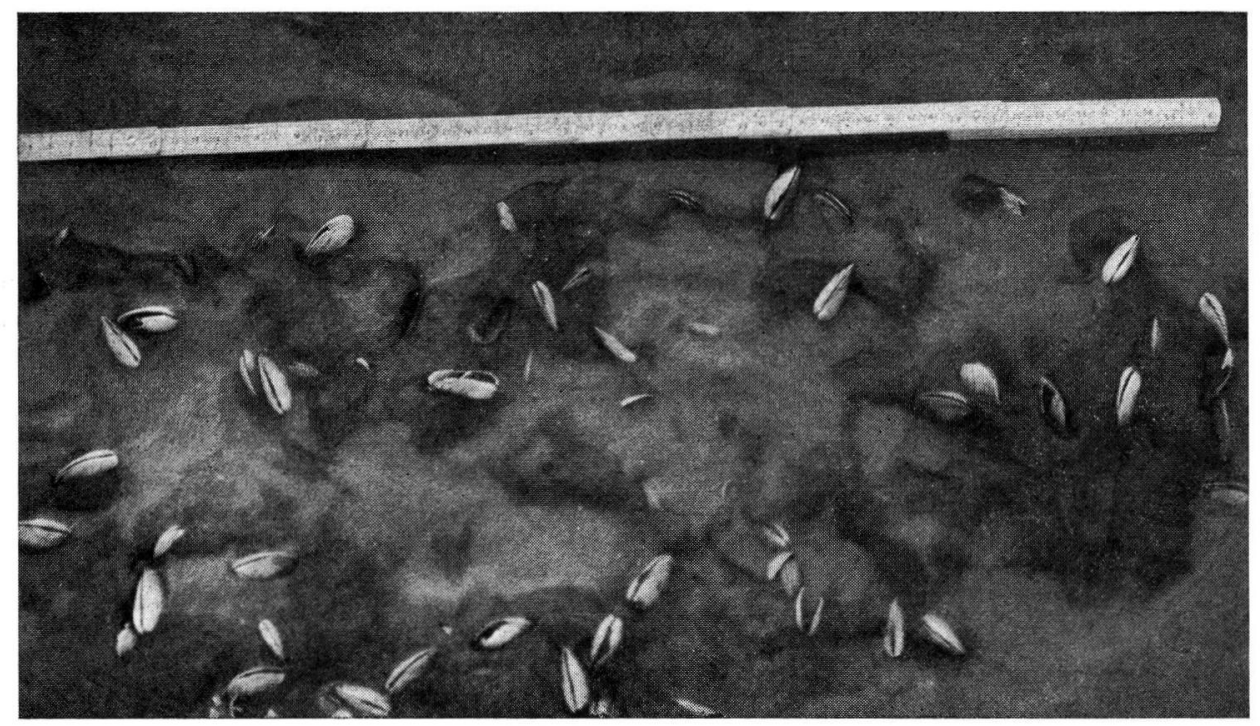

Abb. 3: Flächenhafte Verbreitung von Scrobicularia plana in ehemaligen Schlickwatt-Sedimenten. Unmittelbar östlich der Fundamente des Alten Westturms bei Buhne B.

Die Höhenlage der Probe wurde einnivelliert; sie liegt 0,1 m unter NN. Scrobicularia plana lebt heute im geschützten Schlickwatt knapp unterhalb der mittleren Hochwasserlinie (DöRJEs 1978: Abb. 67). Daraus errechnet sich für die Zeit um 1500 v.h. eine Hochwassermarke, die etwa 1,3 m unter der heutigen lag.

\subsection{Grodenproben vom Nordstrand Wangerooges}

Bei der Sylvester-Sturmflut 1977 war der Strand so stark erniedrigt worden, daß eine alte Grodenschicht im Bereich knapp westlich der Buhne J freigespült wurde (Abb. 1). Da sich Groden auf den Ostfriesischen Inseln nur südlich der Dünenzüge ausbilden, konnte diese Schicht Aufschluß über die Verlagerung Wangerooges nach S geben.

Mit einem neu entwickelten Probenentnahmegerät (Hanisch \& Husemann 1979) wurden insgesamt 10 Profilkerne von bis zu 1,5 m Länge gewonnen (Abb. 4) und sedimentologisch, palynologisch, botanisch sowie radiometrisch untersucht.

Die Sequenz (Abb. 5) beginnt im Liegenden mit einem feinkörnigen, tonigen Sand mittelgrauer Farbe. Er ist hoch bioturbat. Darüber schließt sich ein hellgrauer, schwach toniger Feinsand mit Fein- und Rippelschichtung an, dessen Mächtigkeit zwischen 20 und $40 \mathrm{~cm}$ schwankt. Diese beiden Lagen werden nach ihrer faziellen Ausbildung als alte Wattablagerungen angesprochen. Den Abschluß des Watts bildet eine 2 bis $3 \mathrm{~cm}$ dicke, humose Schicht, die einer ehemaligen Algenmatte entsprechen dürfte. Sie ist sehr mächtigkeits- und niveaukonstant, wird in einigen Profilen jedoch von wenigen $\mathrm{cm}$ Wattsand überlagert.

Darüber liegt hellbrauner Mittelsand, der z. T. stark durchwurzelt ist. Seine Mächtigkeit schwankt im Beprobungsbereich zwischen 40 und $60 \mathrm{~cm}$. Unregelmäßige Schrägschichtung weist auf äolische Umlagerung hin. Es dürfte sich demnach um eine Flugsandlage handeln. 


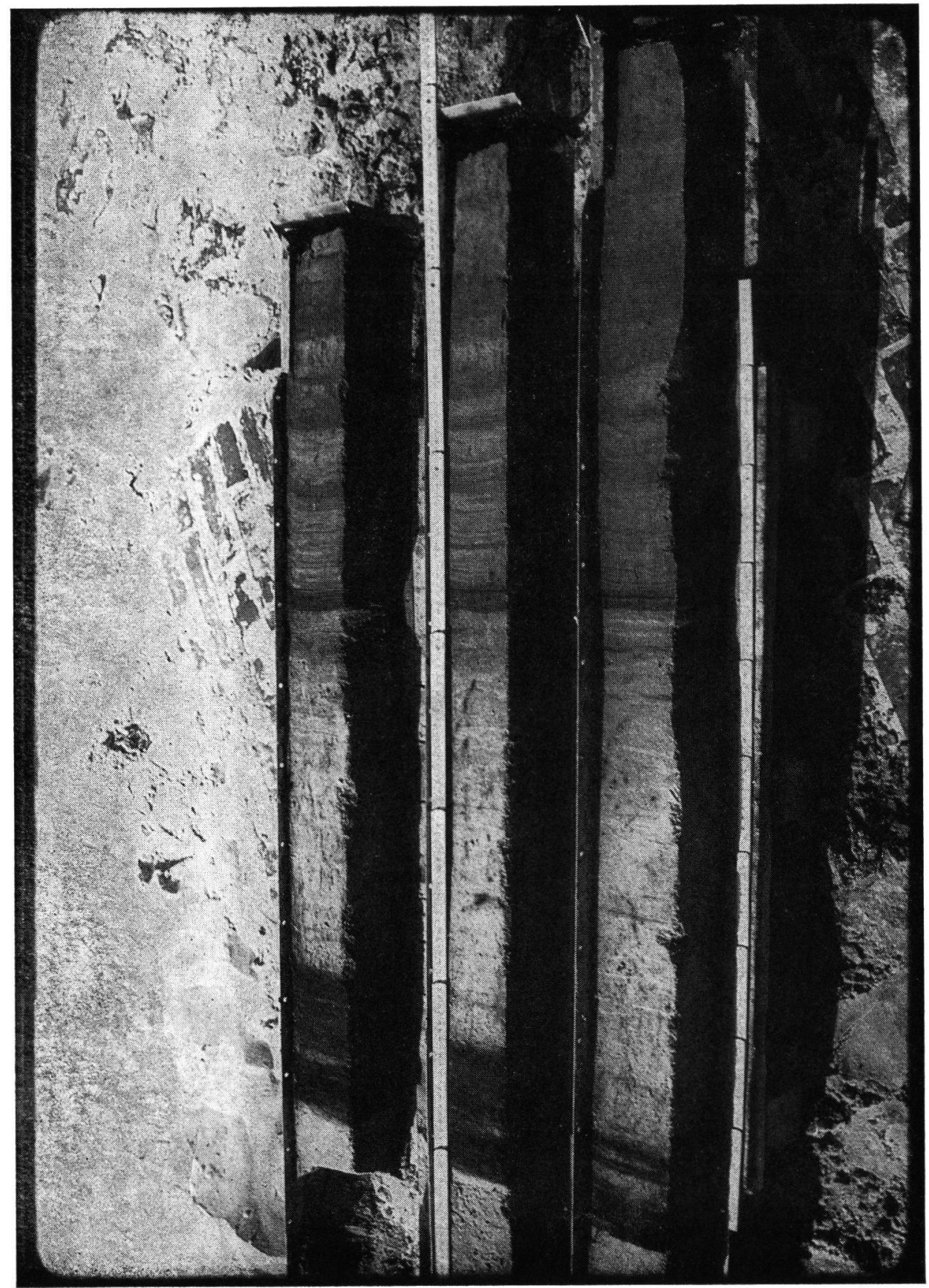

Abb. 4: Drei Kerne mit alten Watt- bis Grodenablagerungen (von rechts nach links). Unter heutigem Strandsand bei Buhne J. 
Den höchsten Teil der Folge bildet eine 10 bis $18 \mathrm{~cm}$ mächtige Schicht stark humosen, tonigen Mittelsands. Lagig sind Wurzel- und Gräserreste (unbestimmbare Graminaen) angereichert. Auch sind millimeterdicke Bändchen von hellgrauem Ton und hellbraunem Mittelsand zwischengeschaltet.

An ausgelesenen Wurzeln und Gräsern wurden ${ }^{14} \mathrm{C}$-Datierungen durchgeführt. Sie brachten Alter von:

$$
\begin{aligned}
& 655 \pm 130 \text { v.h. (Hv 8604) } \\
& 520 \pm 60 \text { v.h. (Hv 8605) } \\
& 580 \pm 80 \text { v.h. (Hv 9169) }
\end{aligned}
$$

Das gemittelte ${ }^{14} \mathrm{C}$-Alter der drei Proben beträgt $555 \pm 45$ v.h.

Aus der gesamten Profilabfolge läßt sich für diesen Zeitraum der Stand des mittleren Hochwassers wie folgt rekonstruieren:

Die Grenze zwischen bioturbaten und geschichteten Wattsedimenten liegt nach RoEP et al. (1975) und Jelgersma (mündl. Mitt.) heute in Holland etwa $40 \mathrm{~cm}$ unterhalb der mittleren Hochwasserlinie.

Algenmatten treten im heutigen Watt Ostfrieslands stets in unmittelbarer Nähe der Hochwassermarke auf.

Die an den Grodenproben durchgeführten, sehr detaillierten Pollenanalysen weisen diese Ablagerung als einen ehemaligen, z. T. weitgehend ausgesüßten „Strandnelkenrasen“ aus. In den obersten Abschnitten sind Anzeichen für Getreideanbau auf der Insel, DünenNähe und Beweidung („feuchte Trittrasen-Gesellschaft“) festzustellen. Nach EllenberG (1963) deutet dies auf ein mittleres Hochwasser hin, das mindestens $25 \mathrm{~cm}$ tiefer als die Groden-Unterkante gelegen hat.

Versucht man diese fossilen Wasserstandsanzeiger mit den heutigen Verhältnissen zu vergleichen, so ergibt sich für das Mitteltidehochwasser, das im Raum Wangerooge heute bei 1,3 m über NN liegt, folgende Situation:

Die Obergrenze der bioturbaten Wattschicht liegt mit $90-95 \mathrm{~cm}$ über NN somit 35 bis $40 \mathrm{~cm}$ unterhalb der mittleren Hochwassermarke.

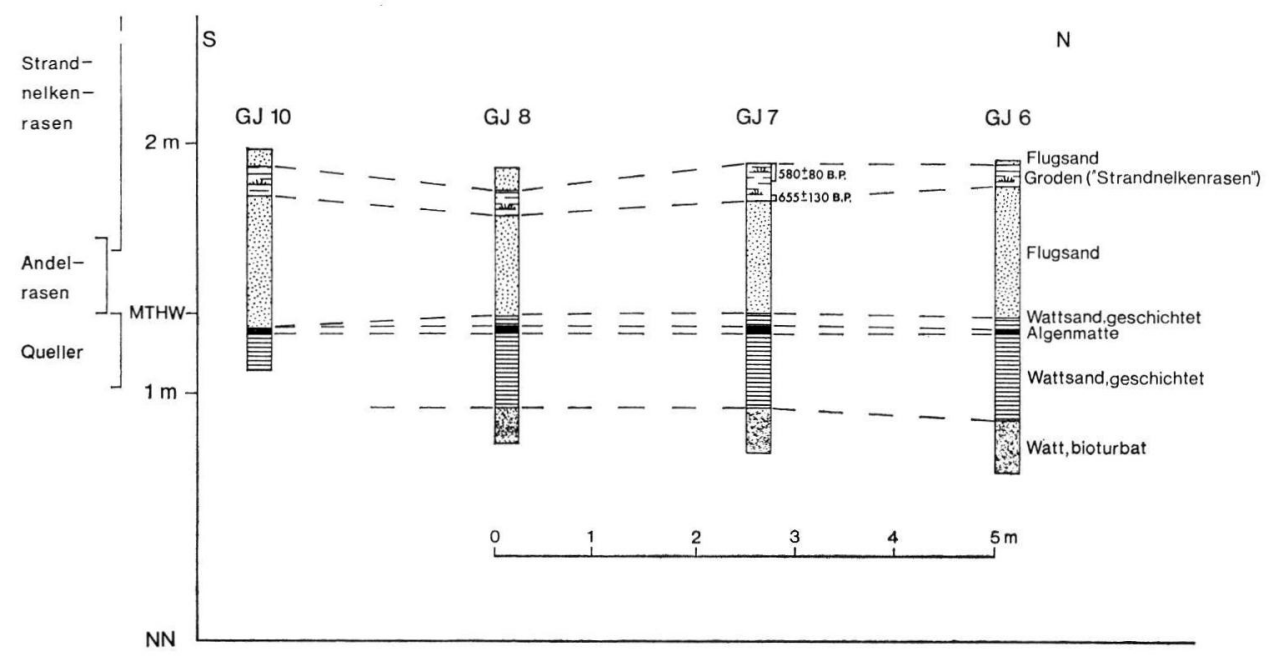

Abb. 5: Höhenlage der alten Watt/Grodenschichten bei Buhne J. 
Die als Algenmatte gedeutete Schicht befindet sich etwa $10 \mathrm{~cm}$ unter MTHW.

Die Grodenlagen befinden sich zwischen 1,70 und 1,95 $\mathrm{m}$ über NN und damit zwischen 40 und $65 \mathrm{~cm}$ oberhalb des MTHW.

Die drei angeführten Höhenmarken befinden sich also in guter Übereinstimmung mit den heutigen Verhältnissen (Abb. 5). Danach muß für die Zeit um 550 v.h. mit praktisch dem gleichen mittleren Hochwasser-Niveau gerechnet werden wie heute. Unter der Annahme unveränderter Tidenhübe hat sich demnach auch der mittlere Meeresspiegel um 550 v.h. auf der gleichen Höhe befunden wie heute. Andererseits ermittelte RoHDE (1977) für die letzten 300 Jahre einen relativen Anstieg des Meeresspiegels um $25 \mathrm{~cm}$ pro Jahrhundert. Aus beiden Befunden wäre demnach für die verbleibende Zeitspanne auf eine kurzfristige Meeresspiegelabsenkung zu schließen.

\section{Schriftenverzeichnis}

DörJes, J. (1978): Das Watt als Lebensraum. - In: Reineck, H.-E.: Das Watt - Ablagerungsund Lebensraum. - 2. Aufl., 185 S.; Frankfurt/M. (Kramer).

Ellenberg, H. (1963): Vegetation Mitteleuropas mit den Alpen in kausaler, dynamischer und historischer Sicht. - In: Walter, H.: Einführung in die Phytologie, 4, 2, 943 S.; Stuttgart (Ulmer).

Hanisch, J. \& H. Husemann (1969): Ein Spül-Stechkasten zur Entnahme ungestörter Sandkerne von 1,5 m Länge. - Senck. marit., 11: 47-57; Frankfurt/M.

Ludwig, G., MülLer, H. \& Streif, H. (1979): Neuere Daten zum holozänen Meeresspiegelanstieg im Bereich der Deutschen Bucht. — Geol. Jb., D 32: 3-22; Hannover.

Roep, Th. B., Beets, D. J. \& RuegG, G. H. J. (1975): Wavebuilt structures in subrecent beach barriers of the Netherlands. - Extr. Publ., 9. Congrès Intern. Sedimentologie, Nice 1975.

Rorde, H. (1977): Sturmfluthöhen und säkularer Wasseranstieg an der deutschen Nordseeküste. Die Küste, 30 : 52-143; Heide/Holst.

Scheer, K. (1953): Die Bedeutung von Phragmites communis Trin. für die Fragen der Küstenbildung. - Probl. Küstenforsch., 5: 15-25; Hildesheim.

Sindowski, K.-H. (1969): Geol. Karte Niedersachsen 1 : 25 000, Erl. Bl. 2213 Wangerooge. 49 S.; Hannover.

Manuskript eingegangen am 24. 1. 1980. 